JGG 2021;69:171-176

doi: $10.36150 / 2499-6564-N 358$

\title{
Multidimensional assessment using a modified S.Va.M.A scale, a pilot study in patients aged 65 or older
}

\author{
Marica Scotellaro', Maila Ruggeri', Victoria d'Inzeo', \\ Emanuele Di Simone ${ }^{2}$, Marco Di Muzio ${ }^{3}$, Sara Dionisi ${ }^{4}$, Noemi Giannetta ${ }^{5}$ \\ ${ }^{1}$ Department of Clinical and Molecular Medicine, Sapienza University of Rome, Rome, Italy; \\ ${ }^{2}$ Nursing, Technical, Rehabilitation, Assistance and Research Department, IRCCS Istituti \\ Fisioterapici Ospitalieri, IFO, Rome, Italy; ${ }^{3}$ Department of Clinical and Molecular Medicine, Sapienza \\ University of Rome, Rome, Italy; ${ }^{4}$ Department of Biomedicine and Prevention, University of Rome \\ Tor Vergata, Italy; ${ }^{5}$ Biomedicine and Prevention - University of Rome Tor Vergata, Italy; Vita-Salute \\ San Raffaele University, Milan, Italy
}

Objective. The progressive demographic increase of the ageing population, with a consequent increase in the average age, is generating important growth in the demand for healthcare services due to the revolving-door and over-crowding phenomena. The aim of this study is to identify the degree of the frailty of the elderly population and the need to activate periodical home assistance in order to counter the aforementioned phenomena.

Methods. A pilot study for adaptation of the SvaMa scale.

Results. The study recruited an overall sample of $n=80$, aged between 65 and 100 years old. Results were analyzed using descriptive statistical analysis, and the Spearman rank correlation coefficient highlights a strong correlation between the different sections of this scale. The data were also analyzed using the Partitioning Around Medoids method, which found that the sample had a good cognitive state but presented functional and mobility issues.

Conclusions. From the data that emerged, we can affirm that the modified S.Va.M.A. scale represents a suitable, fluid, simple instrument to be used whenever a multidimensional assessment of elderly people is necessary

Key words: elderly, homecare, questionnaire, instrumental, predictive

\section{INTRODUCTION}

Italy is currently classified as one of the countries with the highest median age, as the fertility rate is very low, and the average life span tends to lengthen considerably ${ }^{1}$. In 2019, there were about 13.8 million individuals 65 years old or over, or $22.8 \%$ of the entire population, while in 2009 they constituted $20.3 \%$. This phenomenon entails the involvement of every sector, especially the economic one; this is because the need to resort to welfare and health services increases.

A study recently conducted by the Ministry of Economy and Finance ${ }^{2}$ (MEF) reports that there is a strong correlation between health care services consumption with increasing age. Demographic ageing will, in the coming decades, lead to considerable growth in demand for health care goods, in

\footnotetext{
This is an open access article distributed in accordance with the CC-BY-NC-ND (Creative Comm appropriate credit and mentioning the license, but only for non-commercial purposes and only in the original version. For further information: https://creati-

vecommons.org/licenses/by-nc-nd/4.0/deed.en

Copyright by Società Italiana

di Gerontologia e Geriatria (SIGG)
} 
turn generating pressure on the financial system. Precisely for this reason, a difference in the average level of expenditure in the various geographical areas is of great importance. In addition, a particular strengthening of territorial services and a timely and continuous assignment, especially of people with chronic conditions, would lead to fewer complications in the individual and a significant reduction in hospitalizations, counteracting the over-crowding and revolving door ${ }^{3}$ phenomena.

The increase in the incidence of comorbidities and chronic pathologies leads to an increased vulnerability of elderly people developing frailty syndrome, thus creating considerable problems for the public health system ${ }^{4}$.

Frailty has been defined as a "clinical condition characterized by an individual's increased vulnerability to stressors caused by the deterioration of multiple physiological systems" ${ }^{5}$. This geriatric syndrome, established as a predictive index for the development of adverse outcomes and mortality, usually affects individuals that are 65 years and older and aggravates their functions and pathophysiological processes outlined above. The frail elderly are affected by multiple chronic diseases, oftentimes disabled and clinically unstable, which leads to a rapid deterioration of health and excessive consumption of energy. It is a multidimensional condition that, in turn, implies a multi-organ disorder influenced by endogenous and exogenous factors ${ }^{6}$ that cause a homeostatic caducity, so that the elderly present a predisposition to dehydration, hypothermia, hypoglycemia, or a reduction in the functions of self-regulation. In order to counter this situation, it would be necessary to enhance the figure of the family nurse, through careful and continuous home assistance 7,8 . Such a figure would be able to prevent and/or identify acute phenomena at an early stage.

The Health Pact 2019-2021 ${ }^{9}$, in fact, due to the socioepidemiological change, which shows a lengthening of the average life expectancy and an increase in situations of fragility, provides for an enhancement of the health professions, particularly nursing, aimed at covering the needs in the most fragile subjects. In light of the above, Italy would thus be in line with the indications of the World Health Organization (WHO), which had denoted the need for the growth of family/community nurses several years ago ${ }^{10,11}$. We also want to enhance and promote the importance of home care, the fragmentation of which was also demonstrated during the COVID-19 pandemic, with reduced access to care as one of the major obstacles, in turn causing great discomfort in patients with high fragility ${ }^{12}$.

The elderly patient needs to be subjected to a dynamic multidimensional assessment and interdisciplinary process aimed at identifying the physical, mental, and functional nature of a patient who is not self-sufficient. Therefore, the focus of this research work is the elderly population, in particular the frail elderly. The aim of this study is to modify the SVaMA scale (multidimensional evaluation sheet of the elderly person) based on the most significant elements in order to be able to perform a precise and reliable multidimensional assessment of the elderly.

\section{METHODS}

\section{IDENTIFICATION OF THE INSTRUMENT}

To achieve the set objective, the authors analyzed the most recent literature to identify the main scales that are used to assess patients at home aged 65 and over and their degree of predictivity.

The SVaMA ${ }^{13}$ scale has been selected as a suitable study instrument, it is a scale of multidimensional evaluation of the elderly person. In the Veneto Region, it was approved for the first time with the Regional government decision no. 3979 on November 9, 1999, making it possible to evaluate the socio-sanitary conditions of non-self-sufficient elderly people ${ }^{13}$. This scale, in turn, includes other evaluation scales grouped in 4 sheets, in turn, subdivided into 4 sides, which investigate the following aspects:

- the first part is aimed at collecting the person's data, for the assessment of residual potential, the effectiveness of the social network, the autonomy profile;

- the second part is used for the health assessment, in which we relay clinical and pharmacological history, conditions requiring nursing care, sensory and communication analysis and a list of the main pathologies that determine the person's disability;

- the third part deals with cognitive and functional assessment consisting of the following rating scales: Short Portable Mental Status Questionnaire (SPM$\mathrm{SQ})$, Barthel's Index which is divided into Activities Daily Living (ADL) and mobility, and the Exton Smith scale;

- the fourth, and final part, contains spaces for the collection of information on the activation of demand, on the people involved in the assistance, on the enabling, socio-environmental, and economic situations.

\section{Modified SVAMA SCALE}

After careful analysis, it was decided that modifications to the original version of the SVaMA scale were necessary for our study, as such a copious scale could have caused a progressive reduction in patient attention. As to standardize clinical practice, the modified SVaMA score is based on of the most significant elements in 
order to carry out a precise and reliable multidimensional assessment of the elderly ${ }^{14}$ (a copy is available upon request).

The original scale was modified to respect the following adjectives: validity, usability, specificity, and sensitivity. The new SVaMA scale score makes it possible to identify the degree of the frailty of the elderly person and identify the need for periodic home assistance ${ }^{15}$.

The scale is made up of 6 sections: Nursing assistance, Short portable mental status questionnaire, Barthel index for functional situation and mobility, Exton-Smith, and finally the evaluation of communication by Goodclass, Kapla. In turn, these sections have been called: A, B, C, D, E, and F. Subsequently, for each evaluation scale we assigned a score divided into three classes; CLASS 0, CLASS 1, and CLASS 2 which respectively reflect low, medium and high risk. The general research objective is to identify the degree of the frailty of the elderly and to identify the need for home nursing assistance.

\section{PILOT STUDY}

To evaluate the modified version of the instrument, a pilot study was conducted. This research work is a pilot study through which, in compliance with the Declaration of Helsinki and the privacy law, $n=80$ patients were recruited in a hospital in Rome. In addition, all precautions were implemented to ensure that the study respected human rights and that the data collected was processed in aggregate form, therefore not attributable to the respective responders. The study was conducted through a convenience sample, including people over the age of 65 at home or near hospital discharge.

\section{ETHICAL APPROVAL IS REQUIRED IF YOUR STUDY INVOLVES HUMAN OR ANIMAL SUBJECTS}

This pilot study complies with the Declaration of Helsinki and the Privacy Act. All the necessary precautions were taken so that the study respected human rights and the data collected was processed in aggregate form, therefore not attributable to the respective responders.

\section{RESULTS}

\section{StATISTICAL ANALYSIS}

The study recruited a total of $n=80$ patients, of whom $n=38$ men (48\%) and $n=42$ women (52\%); the latter are between 65 and 100 years old, where the arithmetic average is 83.4 , while the standard deviation $(\sigma)$ is +/7.04 (graph 1). Moreover, three-quarters of the cases are in the 75-90 age group (Tab. I), and the score results for each section of the questionnaire are distributed as follows (Tab. II).
Therefore, patients were given the SVaMA questionnaire which included the following sections:

A. nursing

B. cognitive assessment

C. functional assessment

D. mobility assessment

E. prevention - decubitus treatment

F. evaluation of communication

Each item in each section has a score. For each section, an overall score can then be calculated for each patient. In addition, these scores can be grouped together, creating three groups for each section.

Starting from the groupings in Table I for the first $4 \mathrm{sec}$ tions ( $A, B, C, D)$ a table can then be constructed to identify the patient's autonomy profile.

\section{DESCRIPTION OF THE DATASET}

The analysis was conducted on 80 patients, relatively equally distributed by sex (with a slight prevalence of males), between 65 and 100 years of age. Moreover, three-quarters of the cases are in the 75-90 age group (Tab. I), and distributions of the score results for the different sections of the questionnaire are as follows (Tab. II).

It is important to note that for section $\mathrm{A}$, the average score is relatively low compared to the possible range of variation; the latter is in fact between 0 and 16, while the actual scores have a maximum value of 6 . In other cases, the field of variation of the results is almost always equal to or close to the theoretical maximum.

Table I. Distribution of patients by sex and age group.

\begin{tabular}{|l|c|c|c|}
\hline Age class & Woman & Man & Total \\
\hline $65-70$ & 1 & 4 & 5 \\
\hline $71-75$ & 2 & 2 & 4 \\
\hline $76-80$ & 10 & 9 & 19 \\
\hline $81-85$ & 6 & 12 & 18 \\
\hline $86-90$ & 12 & 13 & 25 \\
\hline $91-95$ & 5 & 1 & 6 \\
\hline $96-100$ & 2 & 1 & 3 \\
\hline Total & 38 & 42 & 80 \\
\hline
\end{tabular}

Table II. Distribution of the sum of the different scores in the various sections.

\begin{tabular}{|l|c|c|c|}
\hline Section & Minimal & Maximum & Average \\
\hline A & 0 & 6 & 1,40 \\
\hline B & 0 & 10 & 4,13 \\
\hline C & 0 & 50 & 28,70 \\
\hline D & 0 & 45 & 17,20 \\
\hline E & 6 & 19 & 13,74 \\
\hline F & 0 & 5 & 2,6 \\
\hline
\end{tabular}


Table III. Correlation between the scores of the different sections.

\begin{tabular}{|l|c|c|c|c|c|}
\hline & A & B & C & D & E \\
\hline B & 0,22 & & & & \\
\hline C & 0,41 & 0,52 & & & \\
\hline D & 0,43 & 0,41 & 0,78 & & \\
\hline E & $-0,53$ & $-0,56$ & $-0,77$ & $-0,70$ & \\
\hline F & $-0,26$ & $-0,77$ & $-0,55$ & $-0,44$ & 0,73 \\
\hline
\end{tabular}

The Spearman correlation between the scores of the various sections is presented in Table III.

The greatest link is that between sections $C$ and $D$, i.e. between functional and mobility assessment, which has a correlation coefficient value of 0.78. Exton-Smith's assessment of the risk of bedsores (section E) is also closely related to these two sections, with correlations of 0.77 compared to the functional situation and 0.70 in comparison with mobility. The negative values are due to the fact that the scale of section $E$, like that of section $F$, is such that lower scores correspond to worse situations, unlike in the other sections.

Taking the classification proposed in Table I as a reference, it can be seen that in Section A there are no high-risk patients, while those at medium risk are 11 and those at low risk the majority are 69.

Cognitively (Section B) 10 patients are characterized by severe deterioration, and 20 by moderate deterioration. For the remaining 50 , the deterioration is absent or slight.

Considering the functional situation (Section C), a significant number of patients have issues: 21 patients are fully dependent and 42 partially dependent. Finally, as far as mobility is concerned, 11 patients do not move independently and 26 need some kind of care.

Grouping according to the profile table produced the following results in our dataset: group analysis.

The health profile is a form of patient grouping carried out based on characteristics defined a priori that take into account the scores achieved in sections $A$ to $D$ of the questionnaire.

One question which may be of interest is whether other groupings may emerge from the examination of the data, and how much further consideration of sections $E$ and $F$ of the questionnaire, which does not contribute to the definition of the profile, may affect this.

To try to answer this question, the group analysis technique was used by employing a partitioning around medoids (PAM) partitioning algorithm. In particular, the algorithm was applied both to the dataset consisting of the four variables composed of the scores of sections A, B, C, and D (model 1) and to the dataset made up of the scores of all sections $A$ to $F$ (model 2).
In the use of partitioning algorithms, it is necessary to know a priori the number of groups; in the absence of this information, various sizes can be attempted and the optimum one can be chosen based on predetermined criteria. In our work, the optimal number of groups was chosen on the basis of the silhouette technique and was equal to two in both models.

The interpretation that can be given to the two groups, in both cases, is as follows: group 1 appears to be composed of individuals in need of more assistance than those in group 2 (Tab. IV).

All individuals with a profile of $1,2,7,9$, and 11 are classified in the same groups by the two models; more generally, the differences in group allocation in the two cases concern only six individuals. In almost all (in five) of these, individuals are classified in group 1 using model 1, while they are part of group 2 with the second type of partitioning.

Looking at the individual data, these are in all cases of people who have good health and cognitive situations but have some difficulties from a functional and mobility point of view.

On the contrary, in the only case where the adoption of model 2 has led to the transition from group 2 to group 1 , we have a satisfactory situation concerning mobility and the functional situation but there is serious cognitive deterioration present.

\section{DISCUSSION}

The research objective is to identify the degree of frailty in the elderly and the need to activate periodic home assistance in order to contain acute phenomena that often require hospital admissions, which, in turn, increase the phenomena of revolving-door and overcrowding ${ }^{16}$. Following the descriptive statistical analysis carried out, it emerged that this rating scale allows for the identification and appropriate classification of patients into two groups: those who need more assistance and those who need less assistance. From the sample examined, consisting of $n=80$ patients with an average age of 83.4 years, it was clear that, in this age group, problems related to impaired functionality and mobility were of great importance. Which, in turn, leads to a high risk of injury. From the sample examined, it was clear that problems related to impaired functionality and mobility are of great significance in this age group, a condition that in turn, leads to a high risk of developing pressure ulcers. This conclusion was reached through the application of the Spearman correlation to the study results, which highlighted an important correlation (about 0.77) between sections C, D, $\mathrm{E}$; another evident correlation is that between section $\mathrm{B}$ and F. Furthermore, during the modification phase of the 
Table IV. For each profile value, the number of cases classified in the two groups is highlighted.

\begin{tabular}{|c|c|c|c|c|}
\hline & \multicolumn{2}{|c|}{$\begin{array}{c}\text { Partitioning based on A, B, C, D } \\
\text { (model 1) }\end{array}$} & \multicolumn{2}{c|}{$\begin{array}{c}\text { Partitioning based on A,B,C,D,E,F } \\
\text { (model 2) }\end{array}$} \\
\hline Profile & $\begin{array}{c}\text { Group 1 - more } \\
\text { assistance }\end{array}$ & $\begin{array}{c}\text { Group 2 - less } \\
\text { assistance }\end{array}$ & $\begin{array}{c}\text { Group 1 - more } \\
\text { assistance }\end{array}$ & $\begin{array}{c}\text { Group 2 - less } \\
\text { assistance }\end{array}$ \\
\hline 1 & 0 & 13 & 0 & 13 \\
\hline 2 & 1 & 19 & 1 & 19 \\
\hline 3 & 3 & 8 & 1 & 10 \\
\hline 5 & 5 & 1 & 3 & 3 \\
\hline 6 & 0 & 2 & 1 & 1 \\
\hline 7 & 2 & 6 & 2 & 6 \\
\hline 8 & 11 & 1 & 10 & 2 \\
\hline 9 & 1 & 0 & 1 & 0 \\
\hline 11 & 7 & 0 & 7 & 0 \\
\hline
\end{tabular}

SVAMA scale, as previously mentioned, we considered it necessary to add the communication evaluation scale (section F), which allows us to analyze a fundamental aspect of nursing care. We added sections $E$ and $F$ to the original SVAMA scale profile table which includes the examination of sections $A, B, C, D$, which allowed us to have greater accuracy in determining the degree of assistance needed. Through the Pam type partitioning algorithm, we found that as many as 5 patients passed from a higher degree of assistance to a lower one, and only one patient, on the other hand, passed from lower degree assistance to a greater one. Therefore, to carry out a multidimensional assessment of the patient, it is essential to report section $\mathrm{E}$, which identifies the risk of developing pressure lesions, and section $\mathrm{F}$, which considers the need to resort to augmentative alternative communication techniques in the profile table.

\section{CONCLUSIONS}

The progressive demographic ageing and the continuous occurrence of multiple chronic pathologies has led to particular attention by the research group in identifying an appropriate modus operandi to counter the continuous revolving-door phenomenon. Therefore, this research study aims to identify the degree of frailty and consequently the need to activate periodic home assistance in patients aged 65 years or over. In order to standardize clinical practice, changes were made to the original SVAMA scale, which allowed us to examine a sample of patients recruited at home and close to discharge. Through the modified scale, it was possible to carry out a multidimensional evaluation of the patients and identify which of them needed a greater degree of assistance and which less. As stated, our work focused on making suitable changes that would make the current scale appropriate despite the "weight loss" performed on the original one. The statistical analysis adopted, albeit with a small sample of responders, made it possible to highlight how our scale is adequately investigative in the study of the patient, and above all highlighting the most frequent pathophysiological alterations of a patient aged 65 years or over. Therefore, as concluded by the data that emerged from the descriptive statistical analysis (the study of the correlations between the sections and the analysis of the groups), we can state that the current modified SVaMA scale represents a suitable, fluid, and simple tool to be used in clinical practice whenever a multidimensional assessment of the elderly is necessary. The assessment of the need for home care, which will be periodically carried out by an adequately trained family nurse, will be able to limit the functional decline and improve the quality of life of the frail elderly person and all the people involved.

Therefore, with the presence of a strong territorial care network, multiple beneficial effects can be observed that counteract the phenomena mentioned above and implement the safety of care and the reduction of the risk of error ${ }^{16}$. The importance of promptly and periodically assessing the fragility of the elderly population would lead to a reduction in health services and the costs associated with them ${ }^{17}$.

\section{LIMITATIONS}

Limitations of the study relate to convenience sampling and a relatively small sample size. The study involved a small sample of patients due to the global emergency of COVID-19, which caused the number of hours of clinical training to be reduced, therefore access was contingent and the access to local services was diminished. The study was conducted through a convenience sample and the administration took place in different national areas but still presents a rather modest sampling. 


\section{Ethical consideration}

Pilot study conducted, in compliance with the Declaration of Helsinki and the Privacy Act.

\section{Acknowledgement}

No financial/conflict of interest or source of funding.

\section{Funding}

None.

\section{Conflict of interest}

The Authors declare no conflict of interest.

\section{Author contributions}

Substantial contributions to the conception and design of the work: MS; MR; VD; MDM.

Acquisition, analysis of data: MS; MR; VD; MDM. Interpretation of data: MS; MR; VD; MDM; SD; EDS; NG.

Drafting the work and revising it critically for important intellectual content: MDM; SD; EDS; NG.

Final approval of the version to be published: MS; MR; VD; MDM; SD; EDS; NG.

Agreement to be accountable for all aspects of the work in ensuring that questions related to the accuracy or integrity of any part of the work are appropriately investigated and resolved: MS; MR; VD; MDM; SD; EDS; NG.

\section{References}

1 Istituto Nazionale di Statistica. Anziani: le condizioni di salute in Italia e nell'Unione Europea. Statistiche report, 2017 (https://www.istat.it/it/archivio/203820).

2 Ministero dell'Economia e delle Finanze. II monitoraggio della spesa sanitaria. Roma. Dipartimento della ragioneria generale dello Stato, 2019.

3 Walston J, Buta B, Qian-Li X. Frailty screening and interventions: considerations for clinical practice. Clin Geriatr Med 2018;34:25-38. https//doi.org/10.1016/j. cger.2017.09.004

4 Cammiletti V, Forino F, Palombi M, et al. BRASS score and complex discharge: a pilot study. Acta Biomed 2018;88:414-425. https//doi.org/10.23750/abm. v88i4.6191

5 Gomez-Gomez ME, Zapico SC. Frailty, cognitive decline, neurodegenerative diseases and nutrition interventions. Int J Mol Sci 2019;20:2842. https//doi.org/10.3390/ ijms20112842
6 Waltson J, Robinson TN, Zieman S, et al. integrating fraility research into the medical specialties-report from a U13 Conference. J Am Geriatr Soc 2017;65:2134-2139. https//doi.org/10.1111/jgs.14902

7 Abbasi M, Rolfson D, Khera AS, et al. Identification and management of frailty in the primary care setting. CMAJ 2018;190:e1134-e1140. https//doi.org/10.1503/ cmaj.171509

8 Dionisi S, Di Simone E, Alicastro GM, et al. Nursing summary: designing a nursing section in the electronic health record. Acta Biomed 2019;90:293-299. https//doi. org/10.23750/abm.v90i3.7411

9 Ministero della Salute. Patto della Salute 2019-2021. Roma, 2019 (http://www.salute.gov.it/portale/pattosalute/ dettaglioContenutiPattoSalute.jsp?lingua=italiano\&id=522 2\&area=pattoSalute\&menu=vuoto)

10 World Health Organization. The family health nurse. Contenxt, conceptual framework and curriculum, 2000 (https:// www.euro.who.int/__data/assets/pdf_file/0004/53860/ E92341.pdf).

11 Marcadelli S, Dionisi S. La continuità delle cure. In: Marcadelli S, Obbia P, Prandi C, Eds. Assistenza domiciliare e cure primarie. II nuovo orizzonte della professione infermeristica. Milano: Edra 2018, pp. 195-205.

12 Decreto Legge del 17 marzo 2020, n.18. Misure di potenziamento del Servizio Sanitario nazionale a sostegno economico per le famiglie, lavori e imprese connesse all'emergenza epidemiologica da COVID-19. GU Serie Generale n.70 del 17/03/2020. Roma, 2020.

13 Deliberazione della giunta regionale. Regione Veneto. Assistenza alle persone anziane non autosufficienti. Scheda Svama: profili e livelli di intensità assistenziale, 2007.

14 Marcadelli S, Calò D. Assistenza domiciliare e cure primarie. II nuovo orizzonte delle professioni infermieristica. L'ospedalizzazione a domicilio e in comunità. Milano: Edra 2018, pp. 9-38.

15 Società Italiana di Gerontologia. II problema della ospedalizzazione degli anziani in struttura, 2018 (https://www.sigg. it/news-geriatria/il-problema-della-ospedalizzazione-deglianziani-in-struttura).

16 Pilotto A, Scarcelli C, Addante F, et al. Valutazione multidimensionale dell'anziano fragile. M.D. Medicinae Doctor 2008;27:118-129.

17 Giannetta N, Dionisi S, Cassar M, et al. Measuring knowledge, attitudes and behavior of nurses in medication management: cross-cultural comparisons in Italy and Malta. Eur Rev Med Pharmacol Sci 2020;24:5167-5175. https// doi.org/10.26355/eurrev_202005_21212

18 Angelini S, Alicastro GM, Dionisi S, et al. Structure and characteristics of diabetes self-management applications: a systematic review of the literature. Comput Inform Nurs 2019;37:340-348. https//doi.org/10.1097/ CIN.0000000000000526 